\title{
Should We Routinely Close the Fascial Defect in Laparoscopic Ventral and Incisional Hernia Repair?
}

\author{
Emmanuel E. Sadava, MD, María E. Peña, MD, and Francisco Schlottmann, MD
}

\begin{abstract}
Introduction: Closure of the hernia defect during laparoscopic ventral hernia repair (LVHR) remains controversial. We aimed to analyze whether closing hernia defects impacts in postoperative morbidity and recurrence rates after LVHR.

Materials and Methods: A consecutive series of patients undergoing LVHR from January 2014 to June 2017 with a minimum follow-up of 6 months were included. The sample was divided into two groups: DC, patients with fascial defect closure and NDC, patients without closure of the defect. Postoperative morbidity and recurrence rates were compared between both groups.

Results: A total of 100 patients were included, 51 had their defects closed (DC) and the remaining 49 patients had their defects not closed (NDC). There were no significant differences between groups regarding gender, age, smoking, body mass index, or preoperative American Society of Anesthesiologists. Defect area was similar in both groups (DC: $37 \mathrm{~cm}^{2}$ versus $42 \mathrm{~cm}^{2} \mathrm{NDC}, P=.6$ ). Patients with defect closure had significantly longer operating time (DC: 111 and NDC: 88 minutes, $P<.01$ ). Patients without defect closure showed higher rates of postoperative seroma (DC: $10 \%$ versus NDC: $18 \%$ ) and bulging (DC: $4 \%$ versus NDC: $12.2 \%$ ). After a mean follow-up of 24 (6-36) months, recurrence was 6\% in DC and 18\% in NDC $(P=.07)$. Closure of the defect in midline hernias showed a significant reduction of the recurrence rate (DC: $2 / 39$ [5\%] versus NDC: $5 / 21$ [24\%], $P=.04)$.

Conclusion: Defect closure in LVHR seems to reduce postoperative morbidity and recurrence rates, especially in midline defects. Systematic closure of the hernia defect should be encouraged to improve postoperative outcomes.
\end{abstract}

Keywords: closure defect, laparoscopic, ventral hernia, recurrence

\section{Introduction}

$\mathbf{V}$ ENTRAL HERNIA REPAIR for primary or incisional hernias are common with $>300,000$ cases annually in the United States. ${ }^{1}$ Similar to other minimally invasive procedures, laparoscopic ventral hernia repair (LVHR) has shown several advantages such as fewer postoperative complications, reduced postoperative pain, and shorter length of hospital stay. ${ }^{2,3}$ These benefits have led to a rapid embracement of this surgical approach.

Since its first report by LeBlanc in $1993,{ }^{4}$ the classic technique in which the mesh bridges the hernia defect is likely the most common approach performed by the surgical community. Closure of the hernia defect, however, is an at- tractive alternative because restoring the abdominal wall could improve its function and reduce postoperative complications. ${ }^{5,6}$ Despite these potential benefits, previous studies reflecting no significant reduction in recurrence rates may have discouraged the adoption of this technique. ${ }^{7,8}$ In addition, the 2016 SAGES guidelines for laparoscopic ventral hernia did not make a recommendation regarding defect closure in LVHR due to inconsistent reported data. $^{9}$

Considering that the role of defect closure in LVHR is not yet been elucidated, we aimed to evaluate whether restoring the normal anatomy by closing the hernia defect affects postoperative morbidity and recurrence rates in a single institution.

Division of Abdominal Wall Surgery, Department of General Surgery, Hospital Alemán of Buenos Aires, Buenos Aires, Argentina.

Oral presentation at the American College of Surgeons Clinical Congress, Boston, Massachusetts, October 2018 


\section{Materials and Methods}

After obtaining Institutional Review Board approval, a consecutive series of patients who underwent LVHR between January 2014 and June 2017 in a single institution were analyzed. Patients with size defects between 3 and $8 \mathrm{~cm}$ in width were included for analysis. Patients in whom a technique for relaxing abdominal wall was performed or with a follow-up $<6$ months were excluded.

\section{Surgical technique}

Under general anesthesia, three-port technique was performed. Location of ports was at the discretion of the surgeon and dependent on hernia defect location; for median defects a right-side location was preferred. Once the hernia defect was exposed, the ring was incised by electrocautery to cut the peritoneum and to free the hernia sac. This allows the exposition of the preperitoneal fat at the hernia ring, which is resected to expose the muscle fibers of the ring. Defect measurement was performed intraperitoneally with a ruler and percutaneous spine needles at the borders of the ring.

Defect closure was carried out using nonabsorbable (polypropylene \#0) suture. Running sutures as modified shoelace technique were performed as follows. A loose continuous suture is made and after three or four stitches the end-tail of the thread is passed through the loops. Then, each end of the thread (end-tail and needle-tail) is exteriorized separately with a suture passer and knotted from the outside
(Fig. 1). Depending on the length of the defect, between one and five running sutures would be necessary to close it completely. After closing the whole defect, pneumoperitoneum was evacuated and all sutures were knotted. This technique allows us to reduce skin incisions and operative time.

For prostheses and fixation, bilaminar mesh with viscera protection surface was used in all cases. A $5 \mathrm{~cm}$ overlapping in all directions was used for sizing the mesh, regardless of whether the defect was closed or not. Fixation was performed by transfacial stitches with slowly absorbable material. In addition, mechanical fixation with absorbable tacks in double-crown manner was used.

\section{Patients analysis}

Demographics such as age, gender, body mass index (BMI), smoking, and American Society of Anesthesiologists and operative variables (defect area and operative time) were recorded. Postoperative factors such as hospital stay, seroma, bulging, and recurrence rate were evaluated. The sample was divided into two groups: DC, patients with fascial defect closure, and NDC, patients without closure of the defect. Follow-up evaluation was systematically performed on postoperative days 10-14 and 30, and then every 6 months. Recurrence was detected by either clinical examination or computerized tomography scan (CT scan) when needed. In addition, a CT scan was performed in patients who presented with bulging to rule out a recurrence.
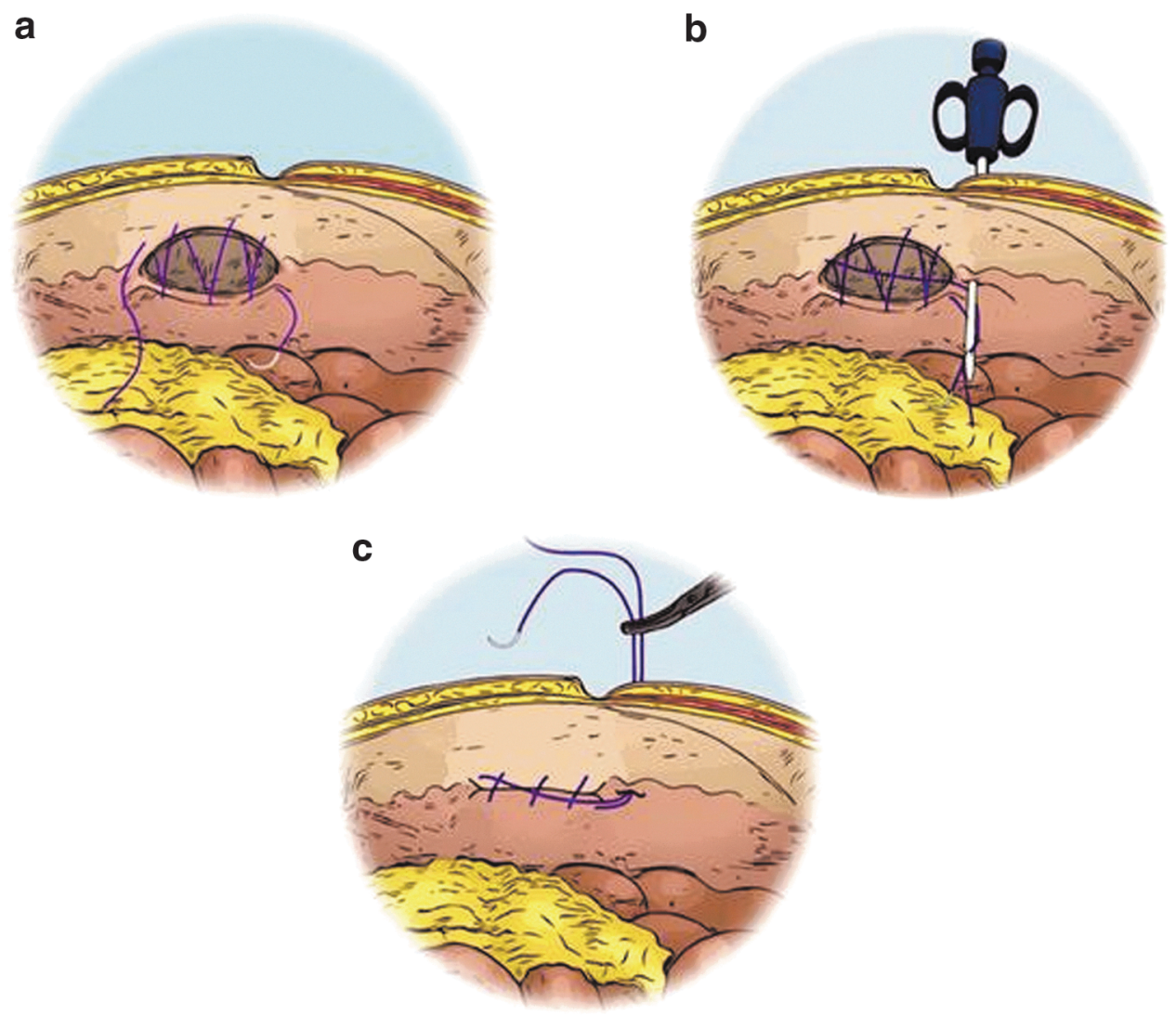

FIG. 1. Modified shoelace technique for closing the hernia defect. (a) A floppy continuous suture is performed. (b) The end tail is passed through the loops, then each tail (end tail and needle tail) is taken out with a suture passer. (c) After closing the whole hernia defect, all sutures are knotted from the outside. 
Table 1. Patients' Characteristics

\begin{tabular}{lccl}
\hline Variable & $\begin{array}{c}D C \\
\mathrm{n}=51\end{array}$ & $\begin{array}{c}N D C \\
\mathrm{n}=49\end{array}$ & $\mathrm{P}$ \\
\hline Age (years) & $63 \pm 14$ & $67 \pm 13.4$ & .08 \\
Gender (male) & $22(43 \%)$ & $27(55 \%)$ & .3 \\
BMI (kg/m $\left.{ }^{2}\right)$ & $27.2 \pm 4.6$ & $26.8 \pm 4.1$ & .3 \\
Smoking (\%) & $8(16)$ & $10(20)$ & .6 \\
Diabetes (\%) & $3(6)$ & $4(8)$ & .7 \\
Hypertension $(\%)$ & $4(8)$ & $3(6)$ & .9 \\
ASA I-II (\%) & $47(92)$ & $47(96)$ & .6 \\
\hline
\end{tabular}

ASA, American Society of Anesthesiologists; BMI, body mass index; DC, with defect closure; NDC, without defect closure.

\section{Statistical analysis}

Data extraction was performed using Microsoft Excel 2010. Chi-square test and Student's $t$-test were used appropriately. Statistical analysis was done with SPSS for Windows 24.0 (SPSS, Inc., Chicago, IL). $P$ value $<.05$ was considered significant.

\section{Results}

A total of 100 patients were included in the analysis: in 51 patients the defect closure was performed (DC) whereas in the remaining 49 patients, defects were not closed (NDC). Patients' demographics and presence of comorbidities were similar between both groups (Table 1). The defect area was $37 \mathrm{~cm}^{2}$ in DC and $42 \mathrm{~cm}^{2}$ in NDC $(P=.6)$. Patients with closure of the hernia defect showed longer operative time (DC: 111 minutes versus NDC: 88 minutes, $P<.01$ ). Length of stay was similar between groups with an average of 1 day in DC group and 1.3 days in NDC group, $P=.8$ (Table 2).

Patients without defect closure were more likely to have postoperative seroma (DC: 10\% versus NDC: 18\%) and bulging (DC: $4 \%$ versus NDC: $12.2 \%$ ), although the difference did not reach statistical significance $(P=.4$ and $P=.1$, respectively). Two patients with bulging and significant impaired quality of life underwent reoperation. Both belonged to the nonclosure group. An open Stoppa was performed in both cases with an uneventful postoperative period.

After a mean follow-up of 24 (6-36) months, a recurrence rate of $12 \%$ was detected. Patients without closure of the defect had three more recurrences than those in whom the closure was performed (NDC: $18 \%$ versus DC: $6 \%$ ); however, no statistical significance was obtained $(P=.07)$. When analyzing subgroups of patients with midline defects, closing the defect showed a significant reduction of the recurrence rate (DC: 2 of 39 [5\%] versus NDC: 5 of 21 [24\%], $P=.04$ ). See Table 3.

Table 2. Operative Factors and Hospital Stay

\begin{tabular}{lccl}
\hline Variable & DC & NDC & $\mathrm{P}$ \\
\hline Mean defect area $\left(\mathrm{cm}^{2}\right)$ & $37 \pm 23.3$ & $42 \pm 28.2$ & .6 \\
Mean operative time (minutes) & 111 & 88 & $\mathbf{. 0 1}$ \\
Mean hospital stay (days) & 1 & 1.3 & .8
\end{tabular}

Cases with defect closure had significantly longer operative time (bold value).

DC, with defect closure; NDC, without defect closure.
Table 3. Postoperative Outcomes

\begin{tabular}{lccc}
\hline Variable & DC & NDC & $\mathrm{P}$ \\
\hline Seroma (\%) & $6(12)$ & $9(18)$ & .4 \\
Bulging (\%) & $2(4)$ & $6(12)$ & .1 \\
Recurrence (\%) & $3(6)$ & $9(18)$ & .07 \\
$\quad$ Midline defects (\%) & $2(5)$ & $5(24)$ & $\mathbf{. 0 4}$ \\
Off midline defects (\%) & 0 & $4(14)$ & .3 \\
\hline
\end{tabular}

Closing midline defect in LVHR showed a significant decrease in recurrence rate (bold value).

DC, with defect closure; NDC, without defect closure; LVHR, laparoscopic ventral hernia repair.

\section{Discussion}

Although LVHR has gained acceptance since its introduction, several modifications have been proposed to improve outcomes. Despite the "standard" LVHR without defect closure confronts the surgical principles of ventral hernia repair, this technique is the most frequently performed. In this study, we aimed to evaluate whether closing the hernia defect during LVHR affects postoperative morbidity and recurrence rates. We found that patients without hernia closure tended to have worse postoperative outcomes and higher recurrence rates, especially in those with midline defects.

In the past years, management of the hernia defect in LVHR has been under debate. Abdominal wall restoration is indeed one of the main principles of ventral hernia repair. Closing the defect augments mesh contact with the abdominal wall attaining a more stable repair with potential fewer postoperative complications. In 2004, Franklin et al. ${ }^{10}$ observed a considerable reduction of recurrence rate after LVHR with defect closure. They found $2.9 \%$ of recurrence rate after an average of 47 months of follow-up. Similarly, in a study of 1326 patients by Chelala et al., ${ }^{11}$ a reduction in overall postoperative complications was observed, and a recurrence rate of $4.7 \%$ was found. Despite being valuable and large researches support of defect closure, no comparative analysis against LVHR without defect closure was performed in those studies. In contrast, a recent meta-analysis that enrolled 16 studies showed that the defect closure during LVHR reduces significantly the total surgical site events. ${ }^{8}$ However, the authors performed a pooled analysis including various types of eventrations with a tremendous heterogenicity, which could bias the results.

In our cohort, recurrence rate was threefold more frequent in patients without defect closure (18\% versus $6 \%)$ and it probably did not reach statistical significance due to our small sample size. A similar trend was observed by Zeichen et al. ${ }^{12}$ with three times more recurrences in the nonclosure group when comparing with defect closure group. In a recent study, closing the hernia defect also showed a significant reduction of the recurrence rate when comparing with nondefect closure. ${ }^{13}$ It is worth to mention that most of the patients in this study were obese with a mean BMI of $37 \mathrm{~kg} / \mathrm{m}^{2}$. Considering obesity as a major risk factor for hernia recurrence, ${ }^{11,14}$ closure of the hernia defect in these patients should be particularly encouraged.

We found that patients with midline defects had significantly different recurrence rates with and without hernia closure (5\% versus 24\%), suggesting that defect closure plays a crucial role in midline defects. Based on Pascal's principle, the abdominal wall is subjected to shear forces, which could 
be stronger at midline. Thus, a restored abdominal wall reinforced by mesh could balance those forces and allow adequate wound healing while enhancing mesh integration. Interestingly, muscle hypertrophy after restoring linea alba has been observed in open repairs but not in laparoscopic bridged repairs. ${ }^{15}$ Those observations highlight the necessity of muscle approximation, thus, restoring the abdominal wall anatomy would provide adequate compliance to balance the shear forces over the time.

Finally, we observed that patients undergoing LVHR with defect closure had fewer postoperative complications. Bulging, also known as pseudorecurrence or mesh eventration, often causes postoperative pain and esthetic complaints. ${ }^{16}$ Assuming that presenting a bulge after LVHR is probably associated with mesh stretching and/or sliding that adapts to hernia sac, the mesh overlapping would be the unique protective factor for hernia recurrence. Thereby, the shear forces at defect edges perpetuate this vicious circle, explaining the discomfort and pain, and potential failures. We observed that patients without defect closure presented bulging three times more frequent (12\% versus $4 \%$ ), and one-third of patients who developed hernia recurrence had history of bulging. These results point out the importance of reducing the dead space by closing hernia defect to improve postoperative outcomes.

This study has several limitations, its retrospective characteristic being the most relevant. In addition, the small sample size probably impacts our results and an increment could have led to major differences between groups. Other factors that have been related to hernia recurrence such as steroids intake, type of meshes, and surgeon's volume were not evaluated. However, we believe that this study is a relevant contribution in the field of ventral hernia repair.

In conclusion, defect closure in LVHR seems to reduce postoperative morbidity and recurrence rates. Systematic closure of the hernia defect should be encouraged to improve postoperative outcomes.

\section{Disclosure Statement}

Drs. E.E.S., M.E.P., and F.S. declare that no competing financial interest exists.

\section{References}

1. Pouluse BK, Shelton J, Phillips S, Moore D, Nealon W, Penson D, Beck W, Holzman MD. Epidemiology and cost of ventral hernia repair: Making the case for hernia research. Hernia 2012;16:179-183.

2. Sauerland S, Walgenbach M, Habermalz B, Seiler CM, Miserez M. Laparoscopic versus open surgical techniques for ventral or incisional hernia repair. Cochcrane Database Syst Rev 2011;2:CD007781.

3. Lomanto D, Ayer SG, Shabbir A, Cheah WK. Laparoscopic versus open ventral hernia mesh repair: A prospective study. Surg Endosc 2006;20:1030-1035.

4. LeBlanc KA, Booth WV. Laparoscopic repair of incisional abdominal hernia using expanded polytetrafluoroethylene: Preliminary findings. Surg Laparosc Endosc 1993;3:39-41.
5. Criss CN, Petro CC, Krpata DM, Seafler CM, Lai N, Fiutem J, Novitsky YW, Rosen MJ. Functional abdominal wall reconstruction improves core physiology and quality-oflife. Surgery 2014;156:176-182.

6. Nguyen DH, Nguyen MT, Askenasy EP, Kao LS, Liang MK. Primary fascial closure with laparoscopic ventral hernia repair: Systematic review. World J Surg 2014;38: 3097-3104.

7. Wennergren JE, Askenasy EP, Greenberg JA, Holihan J, Keith J, Liang MK, Martindale RG, Trott S, Plymale M, Roth JS. Laparoscopic ventral hernia repair with primary fascial closure versus bridged repair: A risk-adjusted comparative study. Surg Endosc 2016;30:3231-3238.

8. Tandon A, Pathak S, Lyons NJ, Nunes QM, Daniels IR, Smart RJ. Meta-analysis of closure the facial defect during laparoscopic incisional and ventral hernia repair. Br J Surg 2016;103:1598-1607.

9. Earle D, Roth JS, Saber A, Haggerty S, Bradley JF 3rd, Fanelli R, Price R, Richardson WS, Stefanidis D; SAGES Guidelines Committee. SAGES guidelines for laparoscopic ventral hernia. Surg Endosc 2016;30:3163-3183.

10. Franklin ME, Gonzalez JJ, Glass JL, Manjarrez A. Laparoscopic ventral and incisional hernia repair: An 11-year experience. Hernia 2004;8:23-27.

11. Chelala E, Baraké H, Estieveneart J, Dessily M, Charara F, Allé JL. Long-term outcomes of 1326 laparoscopic incisional and ventral hernia repair with the routine suturing concept: A single institution experience. Hernia 2016;20:101-110.

12. Zeichen MS, Lujan HJ, Mata WN, Maciel VH, Lee D, Jorge I, Placencia G, Gomez E, Hernandez AM. Closure versus non-closure of hernia defect during laparoscopic ventral hernia repair. Hernia 2013;17:589-596.

13. Martin del Campo LA, Miller HJ, Elliot HL, Novitsky YW. Laparoscopic ventral hernia repair with and without defect closure: Comparative analysis of a single-institution experience with 783 patients. Hernia 2018:22:1061-1065.

14. Vidocvic D, Jurisic D, Franjic D, Glavan E, Ledinsky M, Bekavac-Beslin M. Factors affecting recurrence after incisional hernia repair. Hernia 2006;10:322-325.

15. De Silva GS, Krpata DM, Hicks CW, Criss NC, Gao Y, Rosen MJ, Novitsky YW. Comparative radiographic analysis of changes in the abdominal wall musculature morphology after open posterior component separation or bridging laparoscopic ventral hernia repair. J Am Coll Surg 2014;218:353-357.

16. Sharma A, Mehrotra M, Khullar R, Soni V, Baijal M, Chowbey PK. Laparoscopic ventral/incisional hernia repair: A single centre experience of 1242 patients over a period of 13 years. Hernia 2011;15:131-139.

Address correspondence to:

Emmanuel E. Sadava, MD Division of Abdominal Wall Surgery Department of General Surgery Hospital Alemán of Buenos Aires Buenos Aires C1118ATT Argentina

E-mail: esadava@hospitalaleman.com 